BMJ

Open

Gastroenterology

\title{
Phase II study of neo-adjuvant chemotherapy for locally advanced gastric cancer
}

\author{
Alex Yuang-Chi Chang, ${ }^{1}$ Kian Fong Foo, ${ }^{2}$ Wen-Hsin Koo, ${ }^{2}$ Simon Ong, ${ }^{2}$ \\ Jimmy So, ${ }^{3}$ Daniel Tan, ${ }^{4}$ Khong Hee Lim $^{5}$
}

To cite: Chang AY-C, Foo KF, Koo W-H, et al. Phase II study of neo-adjuvant chemotherapy for locally advanced gastric cancer. BMJ Open Gastro 2016;3: e000095. doi:10.1136/ bmjgast-2016-000095

Received 8 April 2016 Revised 19 May 2016 Accepted 24 May 2016
'Department of Medical Oncology, Johns Hopkins Singapore, Singapore 2Department of Medical Oncology, National Cancer Centre, Singapore

${ }^{3}$ Department of Surgery, National University Hospital, Singapore

${ }^{4}$ Department of Surgery, Alexandra Hospital,

Singapore

${ }^{5}$ Department of Surgery, Tan Tock Seng Hospital, Singapore

Correspondence to Professor Alex Chang Yuang-Chi;

alexchang@imc.jhmi.edu

\section{ABSTRACT}

Background: Neoadjuvant chemotherapy improves survival of locally advanced gastric cancer patients. However, benefit is limited and the best regimen remains controversial.

Objectives: Our primary objective of this prospective, multicenter phase 2 study was to evaluate the pathological complete response rate (PCR) with 2 cycles of docetaxel and capecitabine.

Methods: To be eligible, patients had to have histologically documented gastric cancer, a ECOG performance status 0 or $1, T_{30 r 4} N_{\text {any }} M_{0}$ staging after oesophagogastroduodenoscopy (OGD), endoscopic ultrasound (EUS), CT scan of thorax and abdomen, and negative laparoscopic examination and peritoneal washing. Eligible patients received two cycles of intravenous docetaxel $60 \mathrm{mg} / \mathrm{m}^{2}$ on day 1 and oral capecitabine $900 \mathrm{mg} / \mathrm{m}^{2}$ two times per day from day 1 to day 14 every 3 weeks. We evaluated the response by CT scan and EUS. The patients underwent curative resection with D2 lymphadenectomy subsequently.

Results: 18 patients were enrolled in the study: $66 \%$ were male and the median age was 60 years. 17 patients had T3 disease at diagnosis. There was no pCR noted. 4 patients had a partial response of $22 \%$ (95\% Cl: $7-42 \%$ ), 8 patients had stable disease and 3 patients had disease progression. The median survival was 17.1 months with 3 long-term survivors after at least 3 years of follow-up. The treatment was well tolerated with neutropenia being the most common toxicity. We observed $22 \%$ grade III and $33 \%$ grade IV neutropenia, but no neutropenic fever or death was observed from chemotherapy.

Conclusion: Neo-adjuvant chemotherapy with docetaxel and capecitabine has limited activity against GC. More effective treatment regimens are needed urgently.

Trial registration number: NCT00414271.

\section{INTRODUCTION}

Gastric cancer represents a major global health problem, with nearly 1 million newly diagnosed cases and more than 700000 deaths yearly worldwide. ${ }^{1}$ In Singapore,

\section{Summary box}

Our study is the first trial employing docetaxel and capecitabine as the neo-adjuvant chemotherapy. In locally advanced gastric cancer, we found that two cycles of docetaxel and capecitabine yielded no pathological complete remissions and $22 \%$ partial response, and 15 of $18(83 \%)$ patients underwent R0 with D2 resection. There were three long-term survivors with a median survival of 17.1 months among 18 patients. Thus docetaxel and capecitabine doses used in our trial were comparable, but not more promising, to those reported in the literature, although the regimen was well tolerated.

What is already known about this subject?

- Neo-adjuvant chemotherapy followed by surgery does prolong survival as compared to surgery alone in patients with locally advanced gastric cancer.

- Commonly used regimens include fluorouracil and cisplatin, or epirubicin, cisplatin and fluorouracil.

- No superior or standard therapeutic regimen has been established.

What are the new findings?

- The study showed the limited activity of docetaxel and capecitabine, unlikely to be superior to other available regimens.

- The treatment was very well tolerated; hence, it may be considered as an alternative regimen.

gastric cancer is one of the leading causes of death with over 480 new cases of gastric cancer each year. The outcome in patients with gastric cancer is poor. The 5-year survival rate after apparently curative surgical resection remains $\sim 20 \%$ in Singapore and in many Western countries. This dismal outcome is associated with the advanced stage at presentation. In Singapore, the majority of patients with gastric cancer present with late stage of disease. ${ }^{2}$ Survivability was mainly affected by local recurrence at tumour bed, or lymph node, as well as diffuse peritoneal involvement and distant metastases. 


\section{Summary box}

How might it impact on clinical practice in the foreseeable future?

We pointed out that:

- Once a patient is diagnosed with gastric cancer, the standard staging procedures such as oesophagogastroduodenoscopy (OGD), endoscopic ultrasound, computed tomography scan of abdomen and pelvis, laparoscopic examination of the abdominal cavity with peritoneal washing should be used before surgery.

- Neo-adjuvant chemotherapy should be considered for locally advanced gastric cancer as one of the standard therapies.

- More research are urgently needed to look for more effective regimens; either targeted therapy, immunotherapy or their combination.

- We advocate to use the pathological complete remission rate as one of the measurements of efficacy of neo-adjuvant chemotherapy in gastric cancer.

The mainstay of treatment of gastric cancer is surgery. ${ }^{3}$ However, despite apparent curative resection, relapse is common especially when the tumour is in the advanced stage. Numerous studies have been conducted to evaluate the use of adjuvant therapy. Recently, a multicentre, randomised, controlled trial from the USA did support the use of adjuvant chemoradiotherapy after surgery for gastric cancer, but over $50 \%$ of patients developed relapse despite chemo-irradiation ${ }^{5}$ in that trial. Adjuvant chemoradiotherapy did not become standard therapy in our region initially, as surgical resection for gastric cancer differs significantly between the East and the West. There was a controversy whether patients would benefit from adjuvant treatment after D1 or D2 resection until the most recent trials demonstrated the survival benefit of $\mathrm{S}-1^{6}$ and combination of oxaliplatin and capecitabine ${ }^{7}$ in $\mathrm{D} 2$ resected patients with gastric cancer.

Neo-adjuvant chemotherapy for gastric cancer was first studied for the locally advanced unresectable disease. Some investigators then employed the concept of neo-adjuvant approach for resectable gastric cancers generally with encouraging results. ${ }^{8-11}$ Neo-adjuvant therapy has several potential benefits:

1. Chemotherapy may downstage cancer to enhance resectability.

2. Patients receive immediate chemotherapy without delay from the major surgery; thus, they may reduce micrometastases.

3. Allows investigators to assess the long-term results in patients who had obtained a complete response with neo-adjuvant chemotherapy at the time of surgery.

4. Those patients with rapidly progressive tumours can be identified. Hence, we could spare them from unnecessary exploratory laparotomy.

At the beginning of our study in 2005, neo-adjuvant chemotherapy for gastric cancer was viewed as experimental. It has become the standard treatment since the
MAGIC trial ${ }^{12}$ with epirubicin, cisplatin and 5-FU (ECF), and the FFCD ${ }^{13}$ study with cisplatin and 5-FU (FP) reported superior survival benefit when compared to surgery alone. However, the best regimen remains to be established.

Various neo-adjuvant chemotherapy regimens have been reported in phase II or III trials. In general, the R0 resection rate was $70-80 \%$, pathological complete response (pCR) was $0-11.7 \%$ and median survival was 15-30 months in resectable patients with gastric cancer receiving neo-adjuvant chemotherapy. ${ }^{14}$ Clearly, more effective treatment is urgently needed.

Docetaxel is a taxane derivative, which inhibits the depolymerisation of microtubule and arrests the cells in mitosis. As a single agent docetaxel yielded 17.5-24\% objective response rate. ${ }^{15}$ When it is added to $\mathrm{CF}$ regimen, DCF (docetaxel, cisplatin, 5-FU) has been reported to be superior to $\mathrm{CF}$ at the expense of more severe toxicity. ${ }^{16}$ DCF and its modified regimens has become a common treatment of choice in the USA. In a recent report of a pharmacokinetic study of docetaxel in Singaporeans, $60 \mathrm{mg} / \mathrm{m}^{2}$ every 3 weeks rather than $\geq 75 \mathrm{mg} / \mathrm{m}^{2}$ was suggested as a single agent in patients with cancer ${ }^{17}$ due to decreased clearance of docetaxel.

Capecitabine is an oral fluoropyrimidine and an active drug for treating gastric cancer with a response rate of $20 \%{ }^{18}$ Capecitabine is metabolically activated by thymidylate phosphorylase preferentially in the tumour cells and has been shown to be synergistic with docetaxel which upregulates the level of thymidylate phosphorylase in cancer cells. ${ }^{19} 20$

The combination of these two agents has been used commonly for breast cancer. The initial result of a phase II study of docetaxel and capecitabine for metastatic gastric cancers showed an overall response rate of $60 \% .^{21}$ The current dose regimen in the treatment of breast cancer has used capecitabine at $950 \mathrm{mg} / \mathrm{m}^{2}$ instead of $1250 \mathrm{mg} / \mathrm{m}^{2}$. This dose regimen of capecitabine is associated with reduced side effects (such as hand-foot syndrome, mucositis and diarrhoea) with the same efficacy. ${ }^{22}$ Therefore, we adopt this dose regimen to combine with docetaxel at $60 \mathrm{mg} / \mathrm{m}^{2}$ as a reasonable and safe treatment. Another advantage of this combination is the convenience to the patient, as capecitabine is an oral agent without a need to use a central venous catheter for continuous intravenous infusion of 5-FU. The Randomized ECF for Advanced and Locally Advanced Esophagogastric Cancer 2 (REAL-2) trial by Cunningham et $a l^{23}$ showed that there was no significant difference in terms of progression-free survival (PFS) and response rate in 5-FU versus capecitabine group, as well as cisplatin versus oxaliplatin group. Docetaxel and capecitabine can be delivered as an outpatient regimen during the entire treatment. Therefore, because docetaxel and capecitabine have been shown to have significant single-agent antitumour activity against advanced gastric cancer, synergistic interaction between each other and very promising partial response (PR) rate 
$(60 \%)$, we decided to study this combination in the neo-adjuvant setting. The dose of each drug was chosen based on the consideration of the ethnical difference and also prior experiences in breast cancer treatment. In this study, we intended to give two cycles of this combination preoperatively for locally advanced, nonmetastatic gastric cancer.

Our primary objective of this trial was to determine the complete pathological response rate (pCR) in patients with locally advanced gastric cancer who received neo-adjuvant chemotherapy docetaxel and capecitabine.

\section{METHODS}

This trial was a single-arm, prospective, multicentre, phase II study performed through the Singapore Gastric Cancer Cooperative Group. Patients were accrued from September 2005 to May 2008. We wanted to report the frequency of long-term survival; therefore, there is a delay in reporting. The protocol was formally approved by the Institutional Review Board of all the participating hospitals and all patients provided informed consent.

\section{Eligibility criteria}

Patients must have endoscopic ultrasound (EUS) staged $\mathrm{T}_{3-4} \quad \mathrm{~N}_{\text {any }} \mathrm{M}_{0}$ gastric (GC) or gastroesophagocardia (GEC) adenocarcinoma in order to be eligible. They must also have negative metastases in the abdomen cavity by laparoscopic examination and peritoneal washing. They could have either initially operable or locally advanced/inoperable disease. GEC tumours had to be Siewert type III lesions. The patients were required to have a Karnofsky performance status $\geq 70 \%$ and adequate haematological, renal, hepatic and pulmonary function. Exclusion criteria included metastatic disease (such as positive peritoneal lavage at staging laparoscopy), prior chemotherapy or radiation therapy, and severe comorbid medical conditions.

\section{Neo-adjuvant chemotherapy}

Treatment consisted of two cycles of induction chemotherapy. The patients received docetaxel $60 \mathrm{mg} / \mathrm{m}^{2}$ in $150-250 \mathrm{~mL}$ normal saline given intraveneously over 1 hour on day 1, while capecitabine was administered at $950 \mathrm{mg} / \mathrm{m}^{2}$ two times per day on days $1-14$. The cycle was repeated every 21 days. Supportive medications included dexamethasone $8 \mathrm{mg}$ two times per day orally for 3 days, starting 1 day prior to docetaxel administration. Antiemetics were administered at the investigator's discretion. Prophylactic growth factor support was not permitted.

A $25 \%$ dose reduction for second cycle was done for grade IV neutropenia lasting $\geq 7$ days, neutropenic fever or grade IV thrombocytopenia from the first treatment. A delay of $\leq 21$ days was permitted prior to the second chemotherapy cycle. Dose delays and reductions were also permitted for non-haematological grade III/IV toxicities.

\section{Surgery}

The patients underwent surgery 3-6 weeks following the last chemotherapy. The extent of gastric resection was dependent on the location of the primary tumour: subtotal gastrectomies were permissible for distal tumours, provided a proximal margin of $\geq 5 \mathrm{~cm}$ could be achieved. Total gastrectomies were indicated for more proximal tumours. The method of reconstruction was at the surgeon's discretion. After a total gastrectomy, a Roux-en-Y esophagojejunstomy was the method of choice. After a subtotal gastrectomy, the gastrojejunostomy could be either a Billroth II type or a Roux-en-Y type.

A D2 dissection of lymph nodes was mandated, that is, all perigastric nodes except the right and left cardial nodes were resected for distal cancers. The decision to perform a more extensive $\mathrm{D} 2$ nodal resection was at the surgeon's discretion.

\section{Adjuvant therapy}

The patients with pathological lymph node involvement following surgery were offered adjuvant chemoradiation, based on the Intergroup 0116 study. ${ }^{5}$ The exact treatment schema or the use of capecitabine versus infusional 5-FU was not specified.

\section{Pre-treatment evaluation and evaluation on study}

Prior to treatment, the patients underwent laboratory evaluation, CT of the abdomen/pelvis, chest X-ray (or CT chest for GEC tumours), esophagogastroduodenoscopy (EGD) with EUS and staging laparoscopy with peritoneal lavage. Upon completing neo-adjuvant chemotherapy, a repeat CT and EUS were performed prior to surgery. When analysing the response in the primary tumour, the results of EUS superseded than those of CT in the event of a discrepancy. Postoperatively, the patients were evaluated every 3 months for the first 2 years, then every 6 months for the next 3 years, then annually. Imaging and testing were carried out as clinically indicated. All toxicities were graded using National Cancer Institute Common Terminology Criteria for Adverse Events, V.3.0.

\section{Statistics}

The primary objective of this study was to estimate the pCR rate to neo-adjuvant chemotherapy by using radiological methods, such as CT scan and EUS. A minimum sample size of 30 patients would permit the pCR rate to be characterised within $\pm 15 \%$. Secondary end points included PFS and overall survival (OS), which were estimated using the Kaplan-Meier method. The pCR was defined as the absence of cancer cells in the resected surgical specimen, including lymph nodes. The OS was defined as the period from the initiation of neo-adjuvant chemotherapy to any cause of death, and the PFS was 


\begin{tabular}{|c|c|c|}
\hline \multirow[b]{2}{*}{ Characteristic } & \multicolumn{2}{|c|}{ Patients $(n=18)$} \\
\hline & No. & Per cent \\
\hline \multicolumn{3}{|l|}{ Age, years } \\
\hline Median & 60 & \\
\hline Range & $47-78$ & \\
\hline \multicolumn{3}{|l|}{ Sex } \\
\hline Male & 12 & 66 \\
\hline Female & 6 & 33 \\
\hline \multicolumn{3}{|c|}{ ECOG performance status } \\
\hline 0 & 8 & 44 \\
\hline 1 & 10 & 56 \\
\hline \multicolumn{3}{|l|}{ Location } \\
\hline Proximal stomach & 5 & 28 \\
\hline Middle stomach & 5 & 28 \\
\hline Distal stomach & 8 & 44 \\
\hline \multicolumn{3}{|l|}{ Staging } \\
\hline T3NX & 1 & 6 \\
\hline T3NO & 6 & 33 \\
\hline T3N1 & 10 & 55 \\
\hline T4NO & 1 & 6 \\
\hline
\end{tabular}

defined as the period from the initiation of neo-adjuvant chemotherapy to the occurrence of an event, recurrence or death, whichever came first.

Analyses were performed on an intention-to-treat basis.

\section{RESULTS}

\section{Demographics}

Eighteen patients were enrolled between May 2005 and May 2008 before the trial was closed by the Research Committee of the Sydney Kimmel Comprehensive Cancer Center of Johns Hopkins University because of slow accrual. Demographics are shown in table 1. The median age of patients was 60 years and most patients $(66 \%)$ were men. Slightly more than half $(56 \%)$ of the tumours were in the proximal and mid-stomach.

\begin{tabular}{lllll}
\multicolumn{6}{l}{ Table 2 } & Chemotherapy toxicities \\
\hline \multicolumn{5}{c}{ Grade* $\%$ of patients) } \\
Toxicity & $\mathbf{1}$ & $\mathbf{2}$ & $\mathbf{3}$ & $\mathbf{4}$ \\
\hline Anemia & $2(11)$ & - & $2(11)$ & - \\
Constipation & $4(22)$ & $1(6)$ & - & - \\
Diarrhoea & $3(17)$ & $4(22)$ & $2(11)$ & - \\
Ml & - & - & - & $1(6)$ \\
Nausea & $2(11)$ & $1(6)$ & $1(6)$ & - \\
Neutropenia & - & - & $4(22)$ & $6(33)$ \\
Alopecia & $2(11)$ & - & - & - \\
Fatigue & $2(11)$ & - & - & - \\
Myalgia & $3(17)$ & - & - & - \\
Insomnia & $2(11)$ & $1(6)$ & - & - \\
Hyperglycaemia & - & $1(6)$ & - & - \\
\hline
\end{tabular}

${ }^{*}$ National Cancer Institute Common Terminology Criteria for Adverse Events, V.3.0.

MI, myocardial infarction.
Chemotherapy delivery and toxicity

Of the 18 patients, 17 (94\%) completed all chemotherapy. One patient developed a myocardial infarction (MI) after the first cycle of chemotherapy and was taken off the protocol. Twenty-two per cent of patients required a dose reduction of capecitabine during the treatment, while $22 \%$ of patients had a dose reduction of docetaxel.

Grade III/IV toxicities and significant grade I/II toxicities observed are listed in table 2. In general, toxicities were consistent with those of the chemotherapy agents. Grade III/IV neutropenia occurred in 10 of the 18 patients $(55 \%)$, but no patient developed neutropenic fever. Grade III nausea and diarrhoea occurred in one $(6 \%)$ and two $(11 \%)$ patients, respectively. No treatment-related death was observed. No mucositis, hand and foot syndrome or transaminitis was reported.

\section{Response to therapy by CT scan and EUS}

Of the 17 patients who completed neo-adjuvant chemotherapy, all underwent repeat CT and EUS but 2 patients' tumours were not measurable by both methods. There were no pCR $(0 \%, 95 \%$ CI $0 \%$ to $18 \%)$. Four of these patients $(22 \%, 95 \%$ CI $7 \%$ to $42 \%)$ were noted to have a PR in the primary tumour, eight patients had stable disease and three patients $(16.7 \%)$ had progressive disease, as shown in table 3 . Three of the four patients who had PR with the neo-adjuvant chemotherapy had a downstaging of their gastric cancer after their surgeries.

\section{Surgery and adjuvant therapy}

One of the 17 patients (6\%) who completed neo-adjuvant chemotherapy developed progressive disease and did not undergo surgery. Another patient was found to have metastatic disease during surgery and underwent a palliative surgical bypass; the patient who developed MI also did not go for surgery. So, 15 patients $(83 \%)$ underwent an R0 resection.

Following surgery, eight patients received adjuvant therapy-four patients were treated with radiotherapy with concurrent fluoropyrimidine (infusional, 5-FU or capecitabine), three patients received fluoropyrimidine alone and one patient was treated with the regimen epirubicin, oxaliplatin and capecitabine (EOX).

Table 3 Response after neo-adjuvant therapy

\begin{tabular}{ll}
\hline Radiological response & $\begin{array}{l}\text { Number of patients } \\
\text { (percentage) }\end{array}$ \\
\hline CR & $0(0)$ \\
PR & $4(22)$ \\
SD & $8(47)$ \\
PD & $3(16.7)$ \\
unmeasurable & $2(11.7)$ \\
\hline CR, complete response; PD, progression of disease; PR, partial \\
response; SD, stable disease.
\end{tabular}




\section{Follow-up and survival}

One patient was lost to follow-up after progression from neo-adjuvant chemotherapy. The rest has been followed up until October 2014. Eleven patients developed recurrent or metastatic disease during follow-up and died of their disease, and three patients were still alive without any evidence of recurrent gastric cancer. The median PFS of the 18 patients was 7 months. The median survival calculated by the Kaplan-Meier method was 17.1 months (7.4 to $>84.7$ months).

Initially, we also planned to study the thymidylate synthetase expression, methylation of RUNX-3 gene ${ }^{24}$ and comprehensive genomic hybridisation ${ }^{25}$ before and after chemotherapy to look for biomarkers of response and prognostic indication. However, due to the lack of pCR and the small number of patients enrolled, we stopped the correlative studies.

\section{DISCUSSION}

We employed two cycles of neo-adjuvant docetaxel and capecitabine in 18 patients with T3 or T4 gastric adenocarcinoma. Although our trial was the first to employ docetaxel and capecitabine as the neo-adjuvant therapy for GC with innovative translational research plans, we closed our study prematurely due to poor accrual. We observed $83 \%$ R0 resection rate, no pCRs and four patients $(22 \%$, $95 \%$ CI $7 \%$ to $42 \%$ ) with PRs. Three patients had progression of disease after neo-adjuvant chemotherapy and the overall median survival was 17.1 months. There were three long-term survivors. Overall, our results are in line with the literature reports and did not appear to be promising. We did not perform any planned correlative laboratory study due to inadequate clinical results.

The strength of our study is in its prospective nature with complete TNM staging of all patients with strict criteria of conducting the trial. Before entry, patients must have CT scan of the abdomen and pelvis (for $\mathrm{N}$ and $\mathrm{M}$ staging), upper endoscopy with EUS (for TNM staging $)^{26-28}$ and laparoscopic examination of peritoneal cavity and peritoneal washing (for M staging). ${ }^{29} 30$

These three procedures are still the standard practice. Laparoscopy is performed to evaluate peritoneal spread when considering whether to give chemotherapy or to do resection first and is indicated for clinical stage $T_{1 b}$ or higher. It is not universally accepted among patients and gastric surgeons, which is one of the causes of poor accrual in our trial. EUS performed prior to any treatment is important in the initial clinical staging of gastric cancer. Patients, who refused EUS, while the initial diagnosis was made by endoscopy, had to be excluded from our study. This also contributes to slow accrual. Currently, the common practice in Singapore for patients with operable gastric cancer is surgery first, as preferred by surgeons and patients, and then followed up by adjuvant chemotherapy. The standard surgical procedure is a subtotal or total gastrectomy with D2 resection. Adjuvant chemotherapy can be either S1 or
oxaliplatin/5-FU/leucovorin. In 2004 or 2005, adjuvant chemotherapy or neo-adjuvant chemotherapy was not uniformly accepted. This might have contributed to slow accrual of our trial. During the course of our study, we learnt the importance of multidisciplinary collaboration and difficulties in conducting clinical trials of gastric cancer. EUS done after chemotherapy or radiation has a reduced ability to accurately determine the posttreatment stage of disease. We understood the limitation of CT scan and EUS as tools for evaluating the response of neo-adjuvant docetaxel and capecitabine in our trial. Therefore, we used pCR as the primary objective of our trial. $^{31}{ }^{32}$ As expected, gastric lesions in the two patients could not be determined radiologically and by EGD post docetaxel and capecitabine.

The low PR rate $(22 \%)$ in our trial might have been accounted by the lower and more tolerable dose of capecitabine $950 \mathrm{mg} / \mathrm{m}^{2}$ and docetaxel $60 \mathrm{mg} / \mathrm{m}^{2}$. A study by Park $e t a l^{21}$ showed that 42 patients with advanced gastric cancer who received 21-day cycles of oral capecitabine $\left(1250 \mathrm{mg} / \mathrm{m}^{2}\right)$ and docetaxel $\left(75 \mathrm{mg} / \mathrm{m}^{2}\right.$ on day 1) had an overall response rate of $60 \%$. The median PFS was 5.2 months and median OS was 10.5 months. Relatively high rate of toxicity was reported, such as grade III hand-foot syndrome $(50 \%)$, neutropenia $(15 \%)$ and leucopoenia (12\%). Hence, Park et al recommended that the dose of capecitabine and docetaxel need to be reduced to 1000 and $60 \mathrm{mg} / \mathrm{m}^{2}$, respectively.

Thuss-Patience et $a l^{33} \quad 34$ investigated the dosedependent efficacy of docetaxel and capecitabine in two patient cohorts with metastatic or recurrent gastric cancer. The first cohort received docetaxel $75 \mathrm{mg} / \mathrm{m}^{2}$ on day 1 plus capecitabine $1000 \mathrm{mg} / \mathrm{m}^{2}$ two times per day on days 1-14 every 3 weeks. The second cohort received docetaxel $60 \mathrm{mg} / \mathrm{m}^{2}$ on day 1 and capecitabine $800 \mathrm{mg} / \mathrm{m}^{2}$ two times per day on days $1-14$ every 3 weeks. The first cohort of patients had a higher response rate $(50 \%$ vs $23.5 \%)$, longer median time to tumour progression (5.6 vs 3.7 months) and survival (10.1 vs 7.2 months). They also noticed more dose reductions were required in the first cohort of patients mainly due to more grade III/IV toxicities. Grade III/IV neutropenia occurred (20\% and $7.8 \%)$ in first and second cohort of patients, respectively. There was a trend in higher response rates with higher doses of docetaxel and capecitabine in other phase II trials. However, our regimen of docetaxel $60 \mathrm{mg} / \mathrm{m}^{2}$ on day 1 and capecitabine $950 \mathrm{mg} / \mathrm{m}^{2}$ days $1-14$ every 3 weeks produced grade III/IV neutropenia of $55 \%$, suggesting that this would be near the maximal tolerated doses without G-CSF support. When added to cisplatin and 5-FU (CF), docetaxel improved the response rate and survival when compared with $\mathrm{CF}$ alone at the expense of more toxicity in patients with metastatic or recurrent gastric cancer. Thuss-Patience et al showed that docetaxel and infusional 5-FU had similar efficacy to the standard ECF. Capecitabine has been shown to have at least equivalent efficacy to infusional 5-FU when given as part 
of a combination regimen in patients with advanced gastric cancer. Several phase II trials have reported promising results with the combination of docetaxel and capecitabine in patients with metastatic gastric cancer. Therefore, it seemed logical to choose docetaxel and capecitabine in the neo-adjuvant setting. Very few studies on the efficacy of docetaxel or other taxane as neo-adjuvant therapy in gastric cancer have been reported. The result of Korean NCIT 01515748 study of docetaxel, oxaliplatin and tegafur as neo-adjuvant chemotherapy is still pending. A German study by Glatz et $a l^{35}$ demonstrated the survival advantage of using

neo-adjuvant and adjuvant chemotherapy, as compared to using neo-adjuvant chemotherapy alone. The chemotherapeutic regimens used were FLOT (docetaxel, folinic acid, fluorouracil and oxaliplatin), ECF (epirubicin, cisplatin and folinic acid) and EOX (epirubicin, oxaliplatin and capecitabine).

Local pharmacogenetics study by Goh et $a l^{17}$ also showed that certain polymorphism in the CYP3A4 gene, the enzyme responsible for the metabolism of docetaxel, was associated with variability in terms of docetaxel clearance from patients' bodies. This study signified that we still have to come up with a better chemotherapeutic regimen that is tailored for the local patient population.

We cannot comment on the HER-2 status on our patients as none had been tested for it, and HER-2 was not a standard test then. HER-2 overexpression indicates poor clinical response and outcome without HER-2 directed therapy.

There are a number of limitations in our trial, which include a small number of patients with wide CIs, premature closure of the trial and delays in reporting as well as lower dose of docetaxel based on the pharmacokinetic and pharmacodynamic study on Singaporeans. We cannot be sure whether higher dose of docetaxel with GCSF (granulocyte colony-stimulating factor) would give better results.

In conclusion, neo-adjuvant chemotherapy with docetaxel and capecitabine at doses used in our study had limited efficacy against locally advanced gastric cancer. More effective and standardised regimens are needed to improve the treatment outcome.

Contributors ACY-C organised the Singapore Gastric Cancer Cooperative Group to run this study, prepared the study protocol and obtained the grant from Singapore Cancer Syndicate. He also prepared the manuscript. FKF, KWH, SO, JS, DT, LKH are members of the Singapore Gastric Cancer Cooperative Group. They contributed to the research idea, actively participated in the research study and enrolled patients in the trial.

Funding This study was partially funded by the Singapore Cancer Syndicate (grant number GN17) to conduct the study. Docetaxel was sponsored by Aventis and capecitabine was sponsored by Roche. Both pharmaceutical companies did not provide any funds to run the study.

Competing interests None declared.

Patient consent Obtained.

Ethics approval National Healthcare Group Review Board (Domain specific review board).
Provenance and peer review Not commissioned; externally peer reviewed.

Data sharing statement No additional data are available.

Open Access This is an Open Access article distributed in accordance with the Creative Commons Attribution Non Commercial (CC BY-NC 4.0) license, which permits others to distribute, remix, adapt, build upon this work noncommercially, and license their derivative works on different terms, provided the original work is properly cited and the use is non-commercial. See: http:// creativecommons.org/licenses/by-nc/4.0/

\section{REFERENCES}

1. Ferlay JSI, Ervik M, Dikshit R, et al. International agency for research in cancer, cancer incidence, and mortality worldwide. Lyon, France: IARC CancerBase no.11, 2013.

2. Ti TK. Pattern and surgical treatment of gastric cancer in Singapore. Br J Surg 1993;807:886-9.

3. Allum WH, Griffin SM, Watson A, et al. Guidelines for the management of oesophageal and gastric cancer. Gut 2002;50 (Suppl 5):v1-23.

4. Nakajima T. Gastric cancer treatment guidelines in Japan. Gastric Cancer 2002;5:1-5.

5. Macdonald JS, Smalley SR, Benedetti J, et al. Chemoradiotherapy after surgery compared with surgery alone for adenocarcinoma of the stomach or gastroesophageal junction. N Eng J Med 2001;345:725-30.

6. Sakuramoto S, Sasako M, Yamaguchi T, et al. Adjuvant chemotherapy for gastric cancer with S-1, an oral fluoropyrimidine. N Eng J Med 2007;357:1810-20.

7. Bang YJ, Kim YW, Yang HK, et al. Adjuvant capecitabine and oxalliplatin for gastric cancer after D2 gastrectomy (CLASSIC), a phase 3 open-label, randomised control trial. Lancet 2012;379:312-15.

8. Ajani JA, Mayer RJ, Ota DM, et al. Pre-operative and postoperative combination chemotherapy for potentially resectable gastric carcinoma. J Natl Cancer Inst 1993;85:1839-44.

9. Earle CC, Maroun J, Zuraw L. Neoadjuvant or adjuvant therapy for resectable gastric cancer? A practice guideline. Can J Surg 2002;45:438-46.

10. Lowy AM, Mansfield PF, Leach SD, et al. Response to Neoadjuvant chemotherapy best predicts survival after curative resection of gastric cancer. Ann Surg 1999;229:303-8.

11. Newman E, Marcus SG, Potmesil M, et al. Neoadjuvant chemotherapy with CPT-11 and Cisplatin downstages locally advanced gastric cancer. J Gastrointest Surg 2002;6:212-18.

12. Cunningham $\mathrm{D}$, Allum WH, Stenning SP, et al. Perioperative chemotherapy versus surgery alone for resectable gastroesophagea cancer. N Engl J Med 2006;355:11-20.

13. Ychou M, Boige V, Pignon JP, et al. Perioperative chemotherapy compared with surgery alone for resectable gastroesophageal adenocarcinoma: an FNCLCC and FFCD multicentre phase III trial. $J$ Clin Oncol 2011;29:1715-21.

14. Biondi A, Lirosi MC, D'Ugo D, et al. Neo-adjuvant chemo(radio) therapy in gastric cancer: current status and future perspectives. World J Gastrointest Oncol 2015;7:389-400.

15. Haller DG, Misset JL. Docetaxel in advanced gastric cancer. Anticancer Drugs 2002;13:451-60.

16. Van Cutsem E, Moiseyenko VM, Tjulandin S, et al. Phase III study of docetaxel and cisplatin plus fluorouracil compared with cisplatin and fluorouracil as first-line therapy for advanced gastric cancer: a report of the V325 study group. J Clin Oncol 2006;24:4991-7.

17. Goh BC, Lee SC, Wang LZ, et al. Explaining interindividual variability of docetaxel pharmacokinetics and pharmacodynamics in Asians through phenotyping and genotyping strategies. $J$ Clin Oncol 2002;20:3683-90.

18. Koizumi W, Saigenji K, Ujiie SK, et al. A pilot phase II study of capecitabine in advanced or recurrent gastric cancer. Oncology 2003:64:232-6.

19. Wagstaff AJ, Ibbotson Tk, Goa KL. Capecitabine: a review of its pharmacology and therapeutic efficacy in the management of advanced breast cancer. Drugs 2003;63:217-36.

20. Maher JF, Villalona-Calero MA. Taxanes and capecitabine in combination: rationale and clinical results. Clin Breast Cancer 2002;2:287-93.

21. Park YH, Ryoo BY, Choi SJ, et al. Phase II study of capecitabine and docetaxel combination chemotherapy in patients with advanced gastric cancer. Br J Cancer 2004;90:1329-33.

22. O'Shanghnessy J. Capecitabine and docetaxel in advanced breast cancer: analysis of a Phase III comparative trial. Oncology (Williston Park) 2002;16(10 Suppl 12):17-22. 
23. Cunningham D, Starling N, Rao S, et al. Capecitabine and oxaliplatin for advanced esophagogastric cancer. $N$ Engl J Med 2008;358:36-46.

24. Li QL, Ito K, Sakakura C, et al. Causal relationship between the loss of RUNX3 expression and gastric cancer. Cell 2002;109:113-24.

25. Tay ST, Leong SH, Yu K, et al. A combined comparative genomic hybridization and expression microarray analysis of gastric cancer reveals novel molecular sub-types. Can Res 2003;63:3309-16.

26. Botet JF, Lightdale CJ, Zauben AG, et al. Endoscopic ultrasound in the preoperative staging of gastric cancer. A comparative study with dynamic CT. Radiology 1991;181:426-32.

27. Bentrem D, Gerdes H, Tang L, et al. Clinical correlation of endoscopic ultrasonography with pathologic stage and outcome in patients undergoing curative resection for gastric cancer. Ann Surg Oncol 2007;14:1853-9.

28. Park SR, Lee JS, Kim CG, et al. Endoscopic ultrasound and computed tomography in restaging and predicting prognosis after neoadjuvant chemotherapy in patients with locally advanced gastric cancer. Cancer 2008;112:2368-76.

29. Sarela Al, Lefkowitz R, Brennan MF, et al. Selection of patients with gastric adenocarcinoma for laparoscopic staging. Am J Surg 2006;191:134-8.
30. De Andrade JP, Mezhir JJ. The critical role of peritoneal cytology in the staging of gastric cancer: an evidence-based review. J Surg Oncol 2014;110:291-7.

31. Lorenzen S, Thuss-Patience P, Al-Batran SE, et al. Impact of pathologic complete response on disease-free survival in patients with esophagogastric adenocarcinoma receiving preoperative docetaxel-based chemotherapy. Ann Oncol 2013;24:2068-73.

32. Homann N, Pauligk C, Luley K, et al. Pathological complete remission in patients with oesophagogastric cancer receiving preoperative 5-fluorouracil oxaliplatin and docetaxel. Int J Cancer 2012;130:1706-13.

33. Thuss-Patience PC, Kretzschmar A, Dogan Y, et al. Docetaxel and capecitabine for advanced gastric cancer: investigating dose-dependent efficacy in two patient cohorts. British $\mathrm{J} \mathrm{Ca}$ 2011;105:505-12.

34. Thuss-Patience PC, Kretzschmar A, Repp M, et al. Docetaxel and continuous-infusion fluorouracil versus epirubicin, cisplatin and fluorouracil for advanced gastric adenocarcinoma: a randomized phase II study. J Clin Oncol 2005;23:494-501.

35. Glatz T, Bronsert $\mathrm{P}$, Schäfer $\mathrm{M}$, et al. Perioperative platin-based chemotherapy for locally advanced esophagogastric adenocarcinoma: postoperative chemotherapy has a substantial impact on outcome. Eur J Surg Oncol 2015;41:1300-7. 